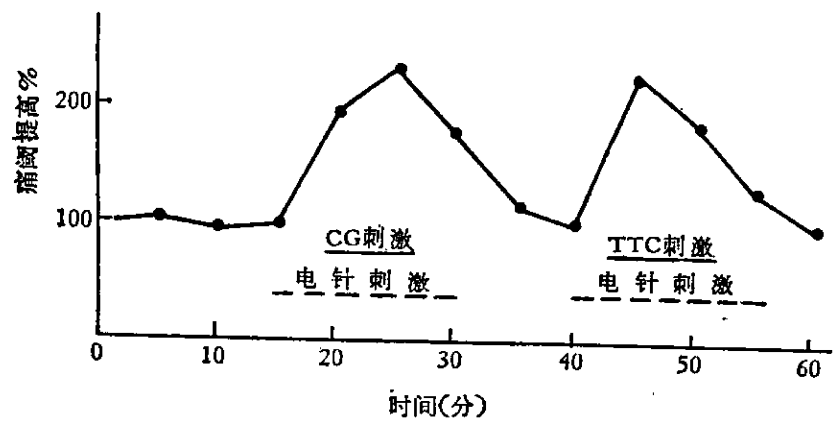

图 4 电针刺激背景上,刺激中央灰质及邻近 被盖对痛阈的影响

针镇痛效应的影响不够明显，但是它并没有 对抗电针镇痛的作用.

\section{参考资 料}

[1] 江振裕等,科学通报, 19 (1974), 1, 31 .

[2] 吴建屏等,科学通报, 18 (1973), 5, 238 .

[3] 上海中医学院等, 中华医学杂志, 1973, 3, 139-
143.

[ 4] 张香桐, 中国科学, 1973, 1, 28一-52.

[ 5 ] Mehler, W. R., Feferman, M. E. and Nauta, W. J. H., Brain, 83 (1960), 718-758.

[6] Nauta, W. J. H. and Kuypers, G. J. M., In Reticular Formation of the Brain, Ed. by Jasper, H. H. et al., Little, Brown, Boston. $1958,3-30$.

[ 7 ] Collins, W. F. and Randt, C. T., J. Neuraphysiol., 23 (1960), 47-53.

[ 8 ] Giuck, H., Becker, D. P. Nulsen, F. E. and Jane, J. A., J. Neurosurg., 30 (1969), 1-13.

[ 9 ] Nakao, H., Yoshida, M. and Sasaki, T., Jap. J. Physiol, 18 (1968), 462-470.

[10] Nashold, B. S., Wilson, W. P. and Slaughter, D. G., J. Neurosurg., 30 (1969), 14-24.

[11] Reynolds, D. V., Science, 164 (1969), 444445.

[12] Mayer, D. J. et al., Science, 174 (1971), 1351-1354.

[13] Sawyer, C. H., Everett, J. W. and Green, J. D., J. Comp. Neurol., 101 (1954), 801-824.

\title{
肉書杆菌毒素阻遏的大鼠神经肌肉 接头在降温后传递功能的恢复
}

\author{
施玉樑沈克飞 \\ (上海生理研究所)
}

肉毒杆菌毒素对胆碱能神经纤维传递的 阻遏作用被认为是不可逆的，毒素与其受体 的结合早在潜伏期的初期业已完 成 ${ }^{[1]}$. 迄今 为止，还没有任何一项措施能使离体神经肌 肉标本被肉毒毒素完全阻断传递的接头恢复 功能. 本文报道经 A 型肉毒杆菌毒素处理完 全麻㾇的接头在降低温度后的恢复.

实验用大白鼠膈神经膈肌标本. 标本制 备、保养及中毒条件同前 ${ }^{[2]}$, 标本以含 $\mathrm{A}$ 型肉 毒杆菌毒素的生理溶液(毒力 $1.7 \times 10^{4}$ 小鼠 最小致死量/毫升溶液)处理半小时。用充以 $3 M \mathrm{NaCl}$ 的玻璃微电极做细胞外记录, 微电 极尖端外径 2-3 $\mu$. 电生理观察从标本对间 接刺激不收缩时开始，电反应直接照相或以
生物用电子计算机叠加处理. 收缩描记按资 料 [3] 进行.

结 果

\section{一、收缩描记}

在我们的实验条件下, $37^{\circ} \mathrm{C}$ 时标本从用 A 型肉毒素处理到在记纹鼓上描记不到收缩 一般约经 60-70 分钟. 此时若给标本施以 间接重复刺激 (100 次/秒一-300 次/秒) 肌肉 仍能出现收缩, 但随着毒素作用的发展, 麻㾇 加深，不久这种对重复刺激的收缩反应也完 全消失. 此后, 若将标本溶液温度降低 10-

本文 1974 年 6 月 3 日收到. 
$15^{\circ} \mathrm{C}$, 肌肉对重复刺激神经又出现收缩, 收 缩幅度逐渐增大，也可以看到肌肉对间接单 刺激的反应. 如再将溶液温度上升到 $37^{\circ} \mathrm{C}$, 收缩反应又消失 (图 1). 在同一标本上上述 现象可多次往复,但重复的次数越多,恢复所 需要的时间越长, 恢复也越不完全. 即使对 重复刺激完全麻痹后在 $37^{\circ} \mathrm{C}$ 温度下保持 70 分钟之久的接头, 降低温度仍能出现上述恢
复现象.

二、降低温度后神经肌肉接头电活动的 恢复

对经肉毒素处理的标本, 当刺激神经不 再能引起肌肉收缩的时候便可以用细胞外微 电极记到神经末梢动作电位和终板电位. 随 着中毒过程的发展, 二者幅度逐渐减小, 并随 着神经末梢传导冲动能力的丧失而完全消

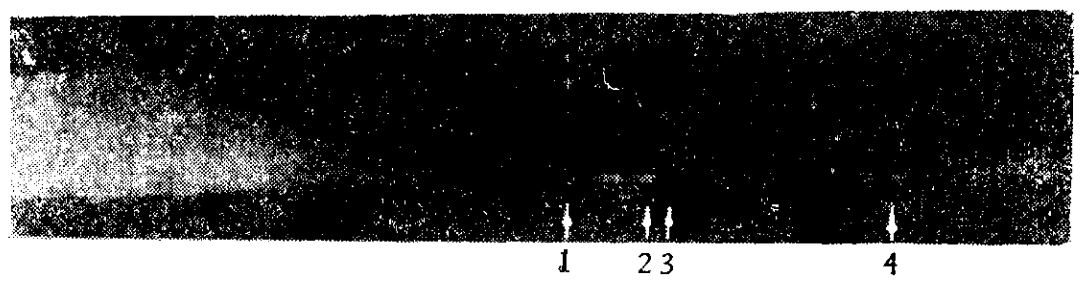

图 1 降温对经肉毒毒素处理麻痹的离体神经肌肉标本的收缩功能的恢复 大鼠膈神经膈肌标本, 在 $37^{\circ} \mathrm{C}$ 下用 $\mathrm{A}$ 型肉莓素处理经 60 分钟记不到单个间接刺激引起的肌肉收 缩. 停鼓半小时, 在箭头 1 处降温至 $20^{\circ} \mathrm{C}$, 标本对单个间接刺激引起的收缩反应有部分恢复. 箭 头 2 处升温到 $37^{\circ} \mathrm{C}$, 对单刺激的反应很快消失, 但对重复刺激仍反应 (箭头 3 处). 待对重复刺激 反应消失后降温至 $20^{\circ} \mathrm{C}$ (箭头 4 处), 对重复刺激又出现反应. 重复刺激每一序列给三炗刺激, 频 率依次为 $50,100,300$ (次/秒)

失 $^{[4]}$. 在消失后立刻或经半至一小时后将标 本溶液温度降低（一般从 $37^{\circ} \mathrm{C}$ 降至 $30-$ $35^{\circ} \mathrm{C}$ ) 可观察到神经末梢动作电位和终板电 位的重新出现 (图 2). 从开始降低温度至反 应恢复的时间可依从完全麻痹到开始降温之

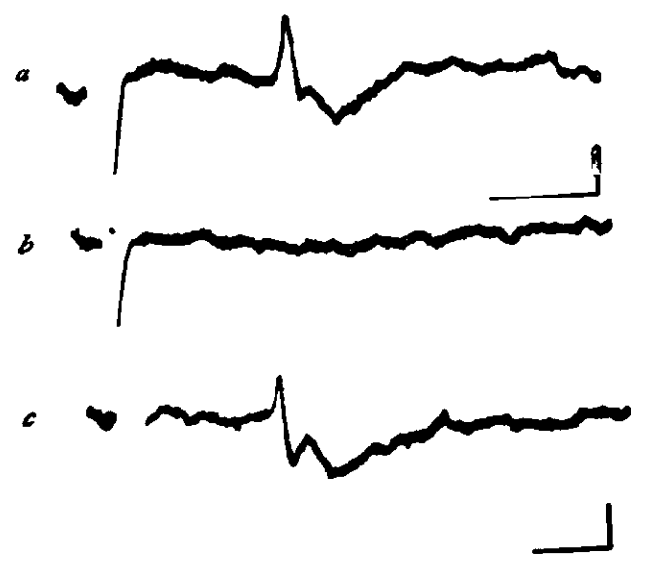

图 2 肉毒中䓯接头的电反应在降温 后的恢复

记录时间及肌槽温度为 $\mathrm{a}-12: 05,34^{\circ} \mathrm{C}$; $\mathrm{b}-12: 15,37^{\circ} \mathrm{C}$ (12:06 升温); c-12:25, $36^{\circ} \mathrm{C}(12: 17$ 降温, 电反应恢复后再升温至 $36^{\circ} \mathrm{C}$, 电反应不立即消失). 电压标记 100 微 伏, 时标 1 毫秒. 大鼠膈神经膈肌标本, 细胞外 记录,电压向上为正,向下为负,以下各图同
间的时间长短而不同: 若对神经末梢动作电 位刚刚消失后的标本降低温度，电反应的恢 复几分钟内即可发生; 若这个时间延长, 甚至 有在降低温度半小时之后才观察到神经末梢 动作电位和终板电位的. 降温后重新出现的 电反应能在此温度下保持, 这时即使再将溶 液温度升高, 电反应也不立即丧失, 虽然从电 反应的潜伏期来看标本温度已升到 $37^{\circ} \mathrm{C}$. 上 面描述的降低温度使神经末梢动作电位和终 板电位重新出现的现象在同一接头可重复多 次, 只是往复的次数越多由降温至反应恢复 所需要的时间越长, 越来越需要降低更大的 温度幅度才出现反应恢复, 而出现的电反应 在低温下保持短时间后也要消失。

电反应恢复之后，还可以观察到在间接 刺激时有些肌纤维恢复收缩 (图 3)，但在降 温后接头电反应恢复的肌纤维不一定都能记 到肌肉动作电位, 特别是对于那些电活动消 失后在 $37^{\circ} \mathrm{C}$ 温度下保留的时间比较长或经 升降温度多次反复的标本.

对电反应已消失的接头降低温度, 神经 

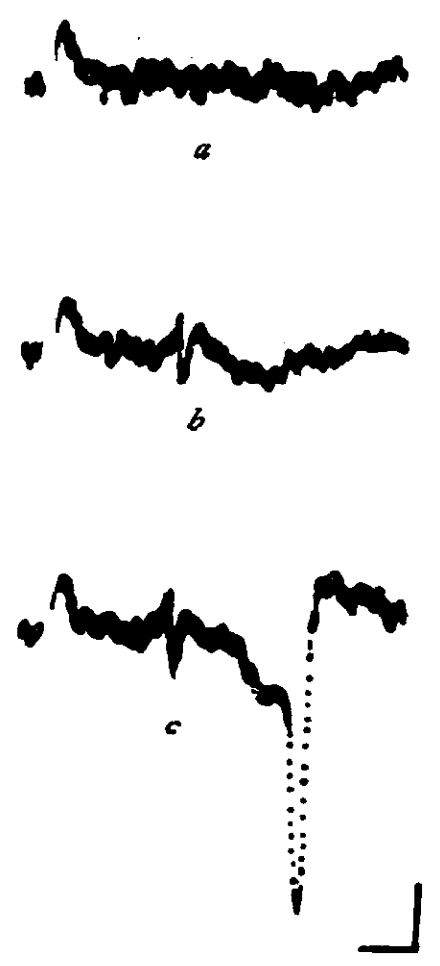

图 3 降温后神经末梢电反应恢 复后肌肉动作电位的重新出现 图中各曲线记录的时间和温度为: $a$ $13: 45,35^{\circ} \mathrm{C} ; \mathrm{b}-14: 30,27^{\circ} \mathrm{C}(14: 00$ 降温); c- $14: 40,26^{\circ} \mathrm{C}$. 电压标记200 微伏. 时标 1 毫秒

末梢动作电位重新出现后其振幅逐步恢复, 与之同时终板电位振幅也逐渐增大. 图 4 表 示降温后的恢复过程. 从 $a$ 到 $e$ 共经 25 分 钟，图中每一条线是经生物用电子计算机将 80 次反应叠加的结果.

讨 论

低温可以延缓肉毒杆菌毒素阻遇接头传 递的时间早已有报道 ${ }^{[1.5]}$, 但本文所描写的降 低温度对肉毒素完全麻㾇接头的恢复作用显

[上接 240 页]

314.7 微米, 最大者为 481.8 微米, 霊壁内分、 中、外三层,各层间的空隙多数较窄, 外壁薄 而易破, 内壁平均厚度为 12.6-13.9 微米(图 版 9), 内壁甚薄.

从当地两例肺吸虫患儿皮下结节中取得 肺吸虫两条（图版 4、5），其形态与获自实验 感染的狗、猫和大白鼠的成虫大致相似，但其

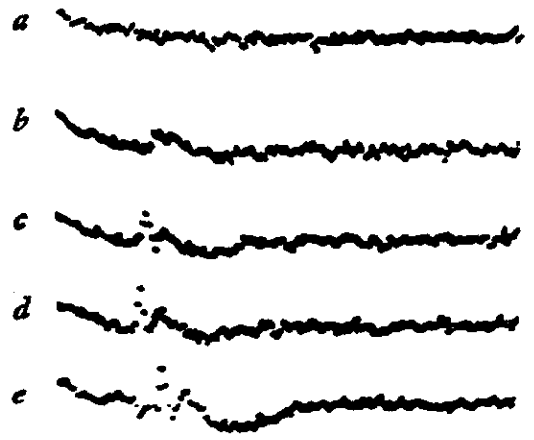

图 4 降温后电反应的恢复过程

反应用电子计算机叠加处理, 每条记录系 80 次反应叠加的结果. 神经末梢电 反应于 19:00 $\left(37^{\circ} \mathrm{C}\right)$ 消失, 随即降温, 各曲线记录 时间与温度为: $a-19: 10,30^{\circ} \mathrm{C} ; \mathrm{b}-$ $19: 15,30^{\circ} \mathrm{C} ; \mathrm{c}-19: 17,30^{\circ} \mathrm{C} ; \mathrm{d}-$ $19: 27,30^{\circ} \mathrm{C} ; \mathrm{e}-19: 35,28.5^{\circ} \mathrm{C}$

然不是一个简单的延缓，而是神经末梢恢复 对兴奋的传导能力，使消失了的神经末梢动 作电位和终板电位在降低温度后的重新出 现. 可以设想, 低温作用于我们现在还不知 道的毒素与其受体作用的某一环节，使其发 生局部的可逆性变化, 恢复神经末梢的传导 能力,末梢和终板电反应重新出现; 但它不能 推动整个过程完全可逆, 所以仍不能使中毒 标本传递功能完全恢复.

\section{参孝 资 料}

[ 1] Burgen, A. S. V., Dickens, F. \& Zatman, L. J., J. Physiol., 109 (1949), 10-24.

[2] 施玉梁、沈克飞, 科学通报, 20 (1975)，2，97.

[ 3 ] Bülbring, E., Brit. J. Pharmacol., 1 (1946), $38-61$.

[4]施玉樑、沈克飞, 科学通报, 20 (1975), 3, 144.

[5] Simpson, L. L., Neuropharmacology, 10 (1971), 673-684.

卵巢及睪丸发育甚差, 子宫内也无虫卵; 这说 明会同肺吸虫对人有致病性，但人并非其合 适的宿主.

致谢：本文所报告的拟钉螺系中国科学院北京 动物研究所刘月英同志鉴定. 薢类系该所戴爱云同 志鉴定，北京医学院附属人民医院照相室门凤江同 志协助摄制部分照片，特此一并致谢. 